\title{
HIMEN IMPERFORADO EN PACIENTE PEDIÁTRICO. REPORTE DE CASO.
}

\section{Imperforate hymen in a pediatric patient. Case report}

\author{
Oskar Andrey Oliveros Andrade1, Juan Carlos Dueñas ${ }^{2}$
}

\section{RESUMEN}

Los trastornos congénitos del tracto genital femenino se presentan con una incidencia del: 10 000-20 000 nacidas vivas, tienen un amplio espectro de manifestaciones clínicas, se describe su detección de manera bimodal, con complicaciones que incluso pueden amenazar con la vida, sin embargo, con un tratamiento oportuno las pacientes pueden tener un buen pronóstico. Se reporta el caso de una paciente de 13 años con cuadro de retención urinaria súbita, estreñimiento agudo, sin menarca y características sexuales adecuadas para la edad, en quien se identificó himen imperforado, hematocolpos. Se descartaron otros posibles diagnósticos diferenciales y el manejo definitivo fue himenotomía más himenoplastía. El himen imperforado hace parte de los trastornos más frecuentes del desarrollo de los genitales femeninos, puede tener múltiples manifestaciones clínicas, leves y severas, con una adecuada identificación, se puede brindar un tratamiento oportuno, y evitar secuelas.

\section{ABSTRACT}

Congenital disorders of female genital tract occurs with an incidence of 1 : $10000-20000$ born alive, have a broad spectrum of clinical manifestations, their detection is described bimodally, with complications that may even threaten life, however, with timely treatment patients can have a good prognosis. We report the case of a I3-year-old patient with a sudden urinary retention, acute constipation, no menarche, and age-appropriate sexual characteristics, in whom an imperforate hymen, hematocolpos was identified. Other possible differential diagnoses were ruled out and the management was hymenotomy plus hymenoplasty. The imperforate hymen is part of the most frequent developmental disorders of the female genitals, it can have multiple clinical manifestations, mild and severe, however, with an adequate identification, it can be given an opportune treatment, good prognosis and avoiding consequences.

\section{INTRODUCCIÓN}

as anomalías congénitas del tracto genital

femenino se presentan con una incidencia de 1:10 000-20 000 nacidas vivas ${ }^{1}$, dentro de sus tipos, el principal es el himen imperforado $^{2,3}$. Pueden ser esporádicos o heredados ${ }^{4}$. La manera de manifestarse clínicamente puede variar ${ }^{5,6}$, y se describe una presentación bimodal ${ }^{7}$. El diagnóstico puede ser realizado en el neonato o en la adolescencia ${ }^{8,9}$. Siempre se deben descartar otros diagnósticos diferenciales $^{10}$, su tratamiento debe ser quirúrgico ${ }^{11} \mathrm{y}$ oportuno para evitar complicaciones.

\section{PRESENTACIÓN DEL CASO}

Se describe el caso de una paciente de 13 años procedente de zona urbana en Cali Colombia, que consulta al servicio de urgencias de un hospital de tercer nivel por cuadro clínico de 2 semanas de evolución consistente en disuria, oliguria, deposiciones cada 2 o 3 días, Bristol tipo 2, y dolor tipo cólico a nivel de hipogastrio, sin fiebre u otro síntoma asociado. Se destaca que la paciente no había presentado menarca ni había tenido su primera relación sexual. Al examen físico, se identificó una masa palpable en fosa iliaca izquierda con sospecha de fecaloma, y adecuadas características sexuales secun-
Residente Pediatría. Fundación Clínica Infantil Club Noel. Universidad Libre Seccional Cali.

Grupo de Investigación en Pediatría (GRINPED COL 0142019)

${ }^{2}$ Cirujano pediatra, Fundación Clínica Infantil Club Noel.

Correspondencia a: Oskar Andrey Oliveros Andrade Correo electrónico: oskaroliveros@hotmail.com Celular: 3217764664

Palabras clave: Trastornos de la menstruación, himen, amenorrea, hematocolpos

Keywords: Menstruation disturbances, hymen, amenorrhea, hematocolpos

Procedencia y arbitraje: no comisionado, sometido a arbitraje externo.

Recibido para publicación: 20 de mayo 2018 Aceptado para publicación: 28 de diciembre de 2018

Citar como:

Rev Cient Cienc Med 2018; 21(2): 37-41 
darias para la edad (Tanner 3). Se solicitó Uroanálisis, Gram de orina y hemograma los cuales fueron normales, en la radiografía de abdomen se evidenciaba abundante materia fecal en colon, se consideró cuadro de constipación, y egresó con hidróxido de magnesio más bisacodilo. Tres días después ingresa nuevamente al servicio de urgencias, con anuria de 24 horas, sin realizar deposiciones desde el alta, dolor tipo cólico en hipogastrio y más intenso, sin inestabilidad hemodinámica ni fiebre, al examen físico una masa palpable en hipogastrio atribuido a globo vesical, la conducta a seguir fue colocación de sonda Foley, donde se evidenció masa en introito de coloración violácea, himen imperforado (ver figura1), la ecografía ginecológica transabdominal solicitada reportó hematocolpos con volumen aproximado de $353 \mathrm{ml}$, sin identificarse otra anomalía congénita del tracto genital femenino. Fue valorada por cirugía pediátrica, se ordenó cateterismos estériles vesicales, analgesia, y el manejo definitivo fue himenotomía más himenoplastía (ver figura 2). Se obtuvo $400 \mathrm{ml}$ de material espeso achocolatado, compatible con menstruación (ver figura 3). Un día después ya con resolución completa de los síntomas se dió egreso de la institución. En sus controles ambulatorios, la paciente realiza deposiciones, diuresis adecuadas, y tiene ciclos menstruales regulares.

\section{DISCUSIÓN}

El himen imperforado es la principal malformación dentro de los defectos de canalización de las malformaciones congénitas del tracto genital femenino ${ }^{2}$, correspondiendo al diagnóstico con el cual contaba nuestra paciente.

Su presentación clínica es variable, puede ser asintomática hasta la menarca o se pueden producir manifestaciones como: amenorrea primaria, siendo esta la más frecuente ${ }^{5}$, dolor abdominal agudo $^{12}$ recurrente, lumbalgia ${ }^{13,14}$, fenómenos obstructivos los cuales afectan el tracto genitourinario, como retención urinaria $^{15}$, mucocolpos en caso de neonatos, y hematocolpos, el cual puede ser observado como una masa que protruye a través de la vagina ${ }^{6}$, siendo un hallazgo encontrado en nuestra paciente y que ya ha sido previamente descrito en la literatura, secundario al himen imperforado y a la retención de menstruación ${ }^{16,17,18}$. Se describe además estreñimiento crónico ${ }^{19}$, obstrucción linfovenosa, masa palpable abdominal debido a la distensión del útero por hematómetra7. La manifestación clínica de retención urinaria secundaria a hematocolpos por himen imperforado ya ha sido documentada en otros reportes de caso ${ }^{5}$. En un paciente se describió incluso retención urinaria con insuficiencia renal aguda ${ }^{20}$, sin embargo, en nuestro caso no se identificó esta complicación. Las manifestaciones clínicas en la paciente, a diferencia de otros casos descritos, fueron el estreñimiento agudo y no crónico, en conjunto a la retención urinaria y hematocolpos, manifestaciones que juntas no se identificaron en otros casos reportados.

El diagnóstico se realiza teniendo en cuenta la historia clínica y el examen físico, y a diferencia de otros casos, en los cuales se diagnostica en la etapa neonatal ${ }^{8}$, en nuestro caso fue realizado a la edad de 13 años. Posterior a su identificación se solicitó una ecografía ginecológica transabdominal en la que se evidenció hematocolpos, y la cual siempre debe realizarse para descartar otras malformaciones del tracto

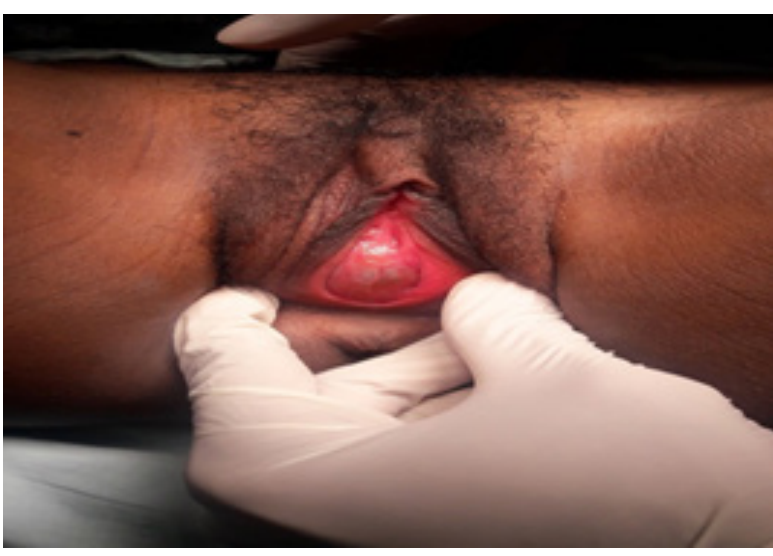

Figura 1: Himen imperforado con hematocolpos.

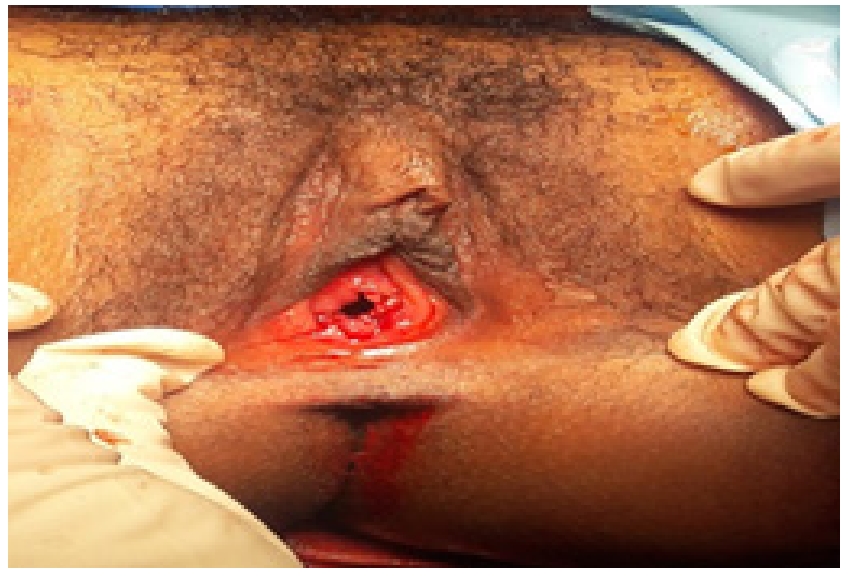

Figura 2: Himenotomía e himenoplastía. 



han sido asociadas y descritas en otros reportes de $\operatorname{casos}^{9}$, sin embargo, en nuestra paciente no se solicitaron estudios imagenológicos adicionales. El diagnóstico de esta anomalía puede ser sospechado desde ecografías obstétricas, y confirmado con el examen físico neonatal, observándose mucocolpos, de no identificarse, éste se puede reabsorber y volver a manifestarse con la llegada de la menarca en la adolescencia ${ }^{3,10}$.

En nuestro paciente se descartaron diagnósticos diferenciales, como: adherencias labiales, septo vaginal obstructivo, quiste vaginal, quiste del ovario, agenesia vaginal, el síndrome de insensibilidad a los andrógenos, prolapso ureteral, ureterocele, quiste parauretral, rabdomiosarcoma vaginal, los cuales ya han sido descritos en la literatura ${ }^{11}$, y no presentó complicaciones como abscesos tubo ováricos ${ }^{21}$ e insuficiencia renal aguda ${ }^{20}$ los cuales ya han sido reportados.

El tratamiento que se realizó fue la himenotomía con incisión en forma de $\mathrm{X}^{2,4}$ entre las 8 y 10 del reloj para disminuir el riesgo de lesión de la uretra, y bajo anestesia, acorde a las recomendaciones mundiales y lo reportado en otros casos $^{22}$. Una recomendación adicional es la no realización de presión sobre el útero para evacuar más sangre durante el procedimiento quirúrgico, dado el riesgo de provocar flujo retró- grado del sangrado hacia la cavidad peritoneal y llevar a endometriosis ${ }^{23}$. La corrección quirúrgica es de buen pronóstico y sin recurrencias ${ }^{24}$.

El diagnóstico de esta patología junto a su abordaje debe ser temprano con el fin de evitar complicaciones debido a la retención de sangre durante la menstruación y compresión crónica o aguda de estructuras adyacentes al útero y vagina, dentro de las mismas descritas en la literatura se destaca: hidroureteronefrosis, falla renal, endometriosis ${ }^{6}$, ruptura uterina ${ }^{5}$, enfermedad pélvica inflamatoria, absceso pélvico ${ }^{25}$, infecciones del tracto urinario, hematosalpinx y hemoperitoneo ${ }^{10}$.

\section{Conclusión}

Se concluye que la manifestación clínica de estreñimiento agudo asociado a retención urinaria en una adolescente con hábitos intestinales y miccionales previos adecuados, deben ser tenidas en cuenta para la sospecha de un fenómeno obstructivo genital, en este caso himen imperforado, por lo que un buen examen físico, acompañado de imagenología adecuada, ayudaría en el diagnóstico y no se debería retrasar su manejo para evitar complicaciones.



Figura 3: Menstruación posterior a himenotomía. 
1. Hořejší J. Congenital developmental defects of derivates of mullerian ducts. Pediatr Adolesc Gynecol Evidence-Based Clin Pract[Internet]. 2012 [Citado 15 de Enero de 2018]; 22:251-70. Disponible en: https://www.karger.com/Article/ Pdf/331689

2. Duyos Mateo I, Abehsera Davó D, De la Calle Fernández-Miranda M, Puch Botella M, del Mar González Arlanzón M. El himen imperforado como urgencia en ginecología: Caso clínico y revisión de la literatura. Progresos Obstet y Ginecol[Internet].2012[Citado 15 de Enero de 2018];55(9):445-8. Disponible en: https://medes. com/publication $/ 77348$

3. Rendón A, Martínez C, Efter C. Himen imperforado, causa de dolor abdominal agudo y hematocolpos en adolescente: reporte de caso clínico. An Med[Internet]. 2012[Citado 20 de Enero de 2018];57:2-5. Disponible en: http:// www.medigraphic.com/pdfs/abc/bc-2012/bc124l. $p d f$

4. Watrowski R, Jäger C, Gerber M, Klein C. Hymenal anomalies in twins-review of the literature and case report. Eur J Pediatr [Internet].2013[Citado 25 de Enero de 2018];173(11):1407-12. Disponible en: https:// link.springer.com/articlel $10.1007 \% 2$ Fs00431013-2123-3

5. Salhan B, Omisore O, Kumar P, Potter J. A rare presentation of imperforate hymen: a case report. Case Rep Urol. 2013 [Internet].2013[Citado el 25 de enero del 2018]; Article ID 731019, 3 pages. Disponible en:http://www.pubmedcentral.nih.gov/ articlerender.fcgi? artid $=3789444 \&$ tool $=$ pmcentrez\&rendertype $=$ abstract

6. Rathod S, Samal S, Rajsekaran A, Rani $\mathrm{P}$, Ghose $\mathrm{S}$. Imperforate hymen and its complications: report of two cases and review of literature. Int J Reprod Contraception, Obstet Gynecol[Internet].2014[Citado 28 de Enero de 2018];3(3):839. Disponible en: https://www. ejmanager.com/mnstemps/ 89/89-1406518342. $p d f$

7. Mwenda AS. Imperforate Hymen - a care cause of acute abdominal pain and tenesmus: case report and review of the literature. Pan Afr Med J[Internet]. 2013[Citado 20 de Enero de 2018];15:2-5. Disponible en: http:// www.panafrican-med-journal.com/content/ article/15/28/full/

8. Ramphul M, Perry L, Bhatia C. Neonatal imperforate hymen with hydrocolpos. BMJ Case Rep[Internet]. 2016[Citado 29 de Enero de 2018];2016:1-2. Disponible en: http:// casereports.bmj.com/content/2016/ bcr-2016215434.full

9. Mustafa B, Deniz U, Cumhur A. Hymen sparing surgery for imperforate hymen: case reports and review of literature. $J$ Pediatr Adolesc Gynecol[Internet]. 2009[Citado 29 de Enero de 2018]; 22 (5): 61-64. Disponible en: https://www.jpagonline.org/article/S10833188(08)00135-6/fulltext

10. Ayaz UM. Ultrasonographic diagnosis of congenital hydrometrocolpos in prenatal and newborn period: a case report. Med Ultrasonography[Internet].2011[Citado 1 de Febrero de 2018];13(3):234-6. Disponible en: https://pdfs.semanticscholar.org//c2493b8f7 d1b16ab3e3ceadfd03dd7ff4b68.pdf

11. González R L, Marín O E, Faíña P V, García D V, Lámelas $\mathrm{P} \mathrm{M}$, Rodríguez $\mathrm{F} \mathrm{V}$, et al. Dolor abdominal como síntoma de himen imperforado. Rev Chil Obstet y Ginecol[Internet].2015[Citado 2 de Febrero de 2018];80(3):261-4. Disponible en: http://www.revistasochog.cl/files/pdf/DRA. LAURAGONZALEZO.pdf

12.Mercado-Alvarado J. Imperforated Hymen: An Unexpected Cause of Pediatric Abdominal Pain,Case Report and Review of Literature. Trop Med Surg. 2013;1(5):10-3.

13. Domany E, Gilad O, Shwarz M, Vulfsons S, Garty BZ. Imperforate Hymen Presenting as Chronic Low Back Pain. Pediatrics. 2013;132(3):e768-70.

14. González J, Matesanz JL, Pablo L De, Duplá B, Fernández B, Meana AR. Caso Clínico Himen imperforado. Revisión casuística a partir de una paciente con dolor lumbar persistente. BOL PEDIATR. 2015; 55: 32-35.

15. González Álvarez CM, García Reymundo M, Real Terrón R, Hawed Ahmed F. Hematocolpos por himen imperforado; causa infrecuente de dolor abdominal y retención aguda de orina. Pediatr Aten Primaria. 2010;12(48):621-6.

16. Makris GM, MacchiellaD, VaidakisD, Chrelias C, Battista MJ, Siristatidis C. Abdominal Tumor 
in a 14-Year-Old Adolescent: Imperforate Hymen, Resulting in Hematocolpos-A Case Report and Review of the Literature. Case Rep Obstet Gynecol. 2015;2015:1-3.

17. Vidaurre MI. Himen imperforado como causa de dolor abdominal en la adolescencia: A propósito de un caso. Arch argentinos. 2014;112(1):2013-5.

18. Fontirroche R, Barroetabeña Y, Rodríguez B, González L. Hematocolpos secundario a himen imperforado en la adolescencia. A propósito de un caso. MediCiego. 2010;16(1):59.

19. Jarabo García MT, García Morán JI. Hematocolpos secundario a himen imperforado. Causa infrecuente de masa abdominal. Rev Pediatr Aten Primaria. 2013;15:339.e165-e168.

20. Papeš D, Arslani N, Rajković Z, Altarac S, Kopjar M. An unusual cause of anuria and hydronephrosis in a 12-year-old girl. Ren Fail. 2011;33(5):540-3.

21. Ho JW, Angstetra D, Loong R, Fleming T. Tuboovarian Abscess as Primary Presentation for Imperforate Hymen. Case Rep Obstet Gynecol. 2014;2014:1-3.Disponible en: doi $10.1155 / 2014 / 142039$

22.Dominguez CE, Rock JA, Horowitz IR.
Surgical conditions of the vagina and urethra. In: Rock JA, eds. TeLinde's Operative Gynaecology. 10th ed. New Delhi: Wolters Kluwer Health/Lippincott Williams \& Wilkins; 2008.p.508-11.

23. Anselm OO, Ezegwui UH. Imperforate hymen presenting as acute urinary retention in a 14-year old Nigerian girl. J Surg Tech Case Rep[Internet]. 2010[Citado 4 de Febrero de 2018];2(2):84-6. Disponible en: https://www. ncbi.nlm.nih.gov/pmc/articles/PMC3214286/

24. Ramareddy, R. S., Kumar, A., \& Alladi, A. (2017). Imperforate Hymen: Varied Presentation, New Associations, and Management. Journal of Indian Association of Pediatric Surgeons. JIAPS[Internet].2017[Citado 7 de Febrero de 2018];22(4):207-10. Disponible en: https://www.ncbi.nlm.nih.gov/pmc/articles/ PMC5615893/

25. Odugu BU OI, Oko DS UI, Onyekpa IJ EP. Imperforate Hymen Presenting with Massive Hematometra and Hematocolpos: A Case Report. Gynecol Obstet[Internet].2015[Citado 8 de Febrero de 2018];5(10). doi:10.4172/21610932.1000328 